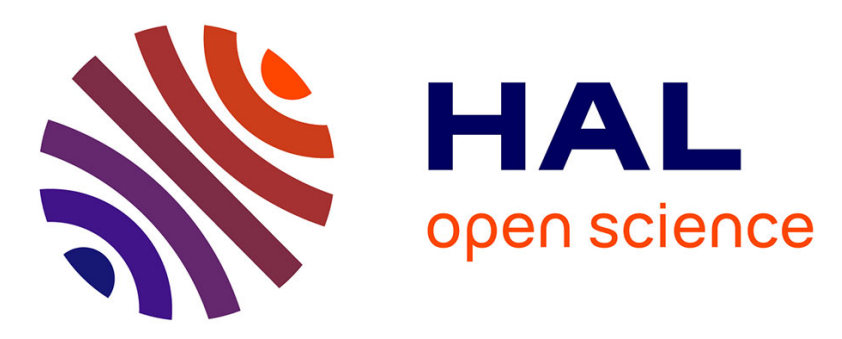

\title{
Interactive Digital Gameplay Can Lower Stress Hormone Levels in Home Alone Dogs - A Case for Animal Welfare Informatics
}

\author{
Annika Geurtsen, Maarten H. Lamers, Marcel Schaaf
}

\section{To cite this version:}

Annika Geurtsen, Maarten H. Lamers, Marcel Schaaf. Interactive Digital Gameplay Can Lower Stress Hormone Levels in Home Alone Dogs - A Case for Animal Welfare Informatics. 14th International Conference on Entertainment Computing (ICEC), Sep 2015, Trondheim, Norway. pp.238-251, 10.1007/978-3-319-24589-8_18. hal-01758456

\section{HAL Id: hal-01758456 \\ https://hal.inria.fr/hal-01758456}

Submitted on 4 Apr 2018

HAL is a multi-disciplinary open access archive for the deposit and dissemination of scientific research documents, whether they are published or not. The documents may come from teaching and research institutions in France or abroad, or from public or private research centers.
L'archive ouverte pluridisciplinaire HAL, est destinée au dépôt et à la diffusion de documents scientifiques de niveau recherche, publiés ou non, émanant des établissements d'enseignement et de recherche français ou étrangers, des laboratoires publics ou privés.

\section{(c)(1)}

Distributed under a Creative Commons Attribution| 4.0 International License 


\title{
Interactive Digital Gameplay Can Lower Stress Hormone Levels in Home Alone Dogs - A Case for Animal Welfare Informatics
}

\author{
Annika Geurtsen ${ }^{1}$, Maarten H. Lamers ${ }^{1}$, and Marcel J. M. Schaaf ${ }^{2}$ \\ ${ }^{1}$ Media Technology Research Group, Leiden University, The Netherlands \\ annika.geurtsen@gmail.com \\ 2 Leiden Institute of Biology, Leiden University, The Netherlands
}

\begin{abstract}
Social isolation, when owners are not home, is a major stressor for dogs leading to separation anxiety and related behavioral and physiological issues. We investigate whether a digital interactive game requiring no human interference reduces stress response in dogs when isolated. An interactive game was developed specifically for canines. Dogs were domestically tested, totaling 15 days with and 15 days without the game. Twice-daily saliva samples were analyzed for cortisol stress hormone concentrations; ethograms were constructed. Combined data confirm that digital interactive games can lessen physiological and behavioral stress responses in dogs, and that the effect is modulated by a dog's personality.
\end{abstract}

Keywords: animal welfare, interactive games, stress, games for health and well-being

\section{Introduction}

Canines are social animals, wired for a life in a pack. However, throughout time, dogs have been domesticated [15] and humans have created artificial living environments for canines (amongst other animals that we domesticated) in which they are required to adapt. The result from living in this artificial environment is that dogs are left alone at home during the day, when their human companions are at work. Social isolation [49] is considered a major stressor for a social species such as canines. Staying home alone, and thus being isolated from the pack, might have an impact on the well-being of the canine [37].

Apart from dogs possibly being bored [29], a considerable number of dogs have developed behavioral issues related to separation anxiety. A recent study conducted for the BBC [11], shows that from a randomized group of $40 \mathrm{dogs}$, at least 10 subjects showed signs of separation anxiety. Moreover, another 25 subjects that did not show anxiety signs, but slept or were lying down while the owners were absent, had cortisol levels that matched those of the dogs showing separation anxiety. Levels of cortisol have been shown to correlate positively with stress experienced by $\operatorname{dogs}[3,4,8,18]$. In other words, even though these 
dogs did not show behavioral signs, they were undergoing stress when they were isolated.

Studies investigating whether human contact reduces stress for shelter dogs show that dogs interacting with humans had lower cortisol levels than dogs that did not interact with humans $[6,44]$. The absence of human presence could allow for a dog to become stressed, and in order to ease the stress, the presence of a human seems required.

Studies into improving the psychological conditions for canines that live in isolation (for several hours every day) using technology is scarce. Products such as SmartDog (Four Legged Trends Ltd., 2014) and PetChatz (Anser Innovation, 2014) focus on strengthening or maintaining the bond between the owner and the dog, by providing direct, live interactions between humans and canines. Providing the canine and owner with the option to initiate contact during separation is a worthwhile aim. However, such solutions circumvent the problem by ensuring the canine is not alone.

Our research embraces the idea that if a digital interactive game is able to entertain the canine sufficiently, the canine may be enabled to become more (emotionally) independent from the human (owner) and as a result might experience less stress when separated from the human. We hypothesize that the presence of a digital interactive game, with which a dog can play without the need of human presence or interference, can reduce the stress response in dogs when left home alone by their owners. This is tested through an experimental study in which we measure cortisol levels in home alone dogs and combine these with video-based ethogram data. We then compare the outcomes across two conditions, namely being at home with and without a digital interactive game specifically designed for canines.

The aims of our research are threefold. Firstly, using intelligent technologies, we aim to improve the quality of life for animals. As such, our work falls within the scope of Animal Computer Interaction (ACI) and Animal Welfare Informatics (AWI) [27]. Secondly, we aim to combine data derived from physiological indicators, i.c. hormonal indicators of stress, and behavioral indicators, i.c. ethograms, to increase the data's reliability in determining a dog's psychological state. This seemingly obvious approach is nonetheless novel within AWI. Thirdly, we aim to widen the application and study of interactive entertainment beyond the human species - a goal that remains compliant with the domain of intelligent technologies for interactive entertainment.

\section{Related work}

Artifacts used for and by animals have been developed in previous decades, but usually not with the intention to specifically improve their welfare. [41]. Since then, the aspirations to serve the aims of animals other than humans in studies have advanced $[27,28]$.

Not all work exploring the relation between technical artefact and animals can be considered AWI. One end of the spectrum focuses on new ways of computing 
using animals, while the other end aims towards using technology to improve the living conditions of the animal. Studies have examined the possibility of animals controlling digital systems (e.g. $[19,22,25,26,42,45,46])$, or the use by animals of a technological medium to communicate with humans (e.g. [20,39]).

An early exploration into AWI is work by Resner [38] which enables owners to clicker train their dog over the Internet. While it is a step towards the positive end of the AWI spectrum, the quality of the interaction is asymmetrical in the sense that the dog merely follows orders, incapable of controlling the application. Training a dog over the Internet supports human needs, but not necessarily those of the canine.

To stimulate both human and animal to participate in interaction one can envision technologically mediated gameplay during which the animal is considered user of the game. The Canine Amusement and Training project [48] allows a dog to join a human in electronic gaming, while simultaneously aiming to motivate the human to spend more time with their canine. As such, it serves the needs of both the canine and human, but the dog remains dependent on the human to initiate gameplay.

A similar approach was undertaken in Cat Cat Revolution [34], a game that allows cats to chase virtual objects on a tablet, a natural behavior of cats. At least partial success of this approach can be deduced from the popularity of Free Games for Cats from Friskies (Nestlé Purina Petcare Company, 2013), tablet games designed for cats and their owners.

The game Pig Chase [14] is an example of an interface that allows the animal to participate as fully recognized user through means of embodied play. The game operates around a large touch-sensitive projection screen set up in a pig stable. On it, pigs can follow small lights with their snout, which are in turn controlled by humans playing on a tablet. Once a pig "catches" a light with its snout, it explodes into a burst of light which according to the makers (among whom animal welfare scientists) pigs enjoy and thus serves as a reward and stimulation. As such it can cognitively stimulate pigs held in captivity and combat its negative effects such as stereotypic behavior development.

Metazoa Ludens [43] is one of few studies where an inter-species game was built, used, and thoroughly evaluated. It allows pet owners to interact and play with their hamster remotely via the Internet in a mixed-reality game. The hamster is placed on an actuated flexible floor and presented moving food that it physically chases and catches. In virtual game space, the human avatar is coupled to the physical food, while being chased by the hamster's avatar. After being allowed over a period of 6 weeks to voluntarily play for one hour per weekday, standardized body condition scoring showed that hamsters' health and fitness had improved. A separate study, aimed at assessing the motivation of the hamsters to play Metazoa Ludens showed that over time the hamster's preference to play increased, allowing a conclusion that the hamsters enjoyed playing the game.

More closely related to our work is Pawtracker [36], an interface that posts sensor-based dog-created content on social media, enabling owners to track their 
dog's activities and share the information with friends. As such, human users know what their pet is up to when home alone. Although the study concerns itself with the issue of dogs being alone at home, it does not directly provide support or a solution for a home alone dog if anxious.

Another study [29] addresses the issue of dogs being left home alone by their owners by providing Internet mediated interactions. For example, the owner is notified when a dog is bored (lying down) and can initiate play remotely by activating a device that throws a ball. Whether this has the desired effect during canine isolation is not investigated. However the study did result in interesting findings on how canines perceive and what their (cognitive and physical) capabilities are in relation to technology.

Although quite a number of related studies exist, the field of ACI is still in its infancy. Co-designing with animals is somewhat of a struggle, and ACI studies cannot make use of certain evaluation methods commonly used in HCI studies, such as surveys. Thus new ways of evaluation must be designed, applied and tested. Although the first exploratory steps were taken, the fields of ACI/AWI will continue to grow, offering valuable insights into the cognitive abilities of animals and resulting design strategies.

\section{Design considerations}

As mentioned above regarding games intended for cats, hamsters and pigs, we suggest that for dogs too, the immersive quality of play could provide a solution to social isolation. In an informal preliminary study we explored design issues for games that potentially prevent canines from getting stressed during isolation. Design choices were assessed informally according to (hypothesized) reactions of canines, feasibility of the game within the constraints of this study, practicality of the game within a domestic setting, and suitability towards the aims of our study.

To successfully design a game for canines, it must adhere to a couple of requirements. Firstly, it must provide suitable stimuli for canine sensory perception $[16,24,33]$. Secondly, the game must comply with canine cognitive capabilities $[7,12,30-32]$, providing a satisfactory challenge to keep dogs immersed. We acknowledge that not all dogs have identical cognitive skills or play style preferences $[10,23]$. The resulting game may not have a similar effect on all dogs, even when capable of performing the same actions. Thirdly, because we aim to provide a game not reliant on human interference, it must work autonomously. Fourthly, [2] dogs do not seem to initiate play when (home) alone or when accompanied by another dog [47]. Therefore, the game must initiate play in order for the dog to immerse in gameplay.

To aid the design process, various commercial dog games were researched and evaluated. Tether Tug (Tether Tug Dog Toys Company, 2013) aims to stimulate individual dog play by appealing to the play action of tugging. A popular product is the Kong (KONG Company), a hollow, rubber object in which food can be placed in the form of treats or foods that can be smeared. Various other com- 
mercial parties produce artefacts designed to mentally stimulate dogs. However, all these games require human presence.

Automation and digitalization of existing games may have the potential of immersing a dog in gameplay that is intuitive and experienced as fun, while also being autonomous, such as GoDogGo Fetch Machine (GoDogGo Inc., 2009) and iFetch (iFetch Company, 2013). Keeping practicalities of our domestically situated experiment in mind, we chose to create a digital interactive game that exploits elements of the electronic game Simon (Milton Bradley Company, 1978), and hide and seek, using sound.

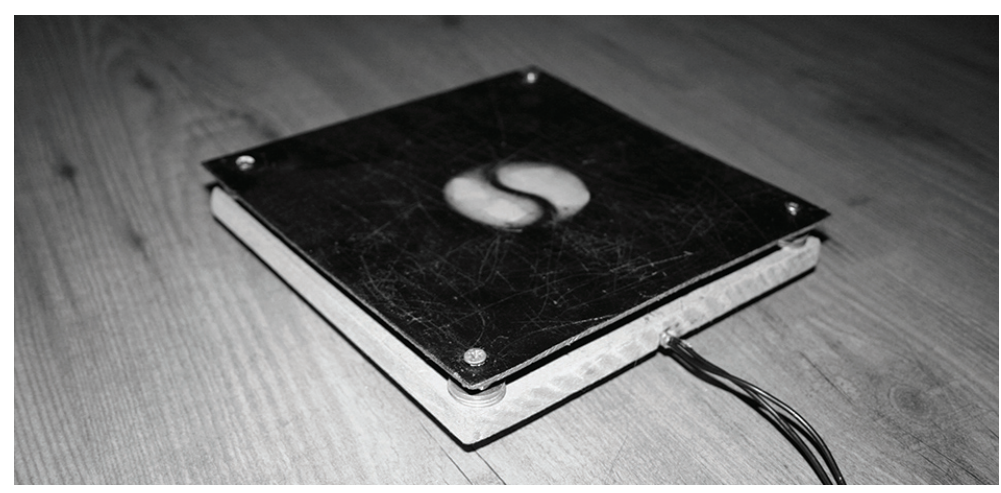

Fig. 1. Button designed specifically to be pressed by canine paws.

\section{The digital interactive game}

The digital interactive game designed for our experiment consists of two game positions, spaced roughly $120 \mathrm{~cm}$ apart, and an electrically controlled dog treats dispenser positioned midway between them. Each game position consists of an audio speaker and button designed specifically to be pressed by canine paws (Fig. 1). The buttons are positioned in front of each speaker, and are basically a microswitch covered by a flexible plastic sheet $(20 \times 20 \mathrm{~cm})$. Placing a paw on the plastic cover depresses the switch, even with minimal force applied - informal tests showed that a weight of 70 grams is sufficient to depress the switch.

The interaction rules are simple and provide direct feedback to aid the dog in understanding gameplay. The game reacts according to the steps-diagram shown in Table 1.

\section{Experimental methods}

\subsection{General setup}

Canine subjects were tested over a total period of 10 days: divided over 5 consecutive weekdays of stimulated condition and 5 consecutive weekdays of unstim- 
Table 1. Game steps diagram

Step Action
1. Wait 20 minutes.
2. From both positions, a position $P$ is randomly selected.
3. Position $P$ emits a short audio sample every 10 seconds, until 2 minutes have
passed or the button at position $P$ was pressed.
4. If button $P$ was not pressed, repeat from step 1.
5. Dispense a treat, and then wait 30 seconds.
6. Alternate position $Q$ emits a short audio sample every 10 seconds, until 2
minutes have passed or the button at position $Q$ was pressed.
7. If button $Q$ was pressed, dispense a treat.
8. Repeat from step 1 .

ulated condition, separated by two days without testing. All testing occurred inside the dogs natural domestic setting. On testing days, the subject was isolated for a set duration, either accompanied by the digital interactive game (stimulated condition) or without it (unstimulated condition). The duration of daily isolation varies per subject (as discussed in more detail later), as does the order in which both conditions were applied. Other animals cohabitating with the subject were excluded from the space wherein the subject was isolated.

\subsection{Salivary cortisol measurements}

Cortisol tests are commonly applied to assess the level of stress experienced by an animal $[3,4,8,18]$. Cortisol can be measured through different sources, such as excretion, blood and hair. We use saliva [10] to provide samples containing cortisol, because differences in cortisol concentration can be measured in saliva within a period of a few hours, whereas cortisol conserved in hair is only detectable over longer periods of time. Furthermore, saliva can be collected at any moment in time, whereas excretion would have to appear during every test within the set timeframe, which is unlikely to happen within a domestic setting. Taking blood samples is intrusive and might itself have an effect on the psychological state of the dog.

Sample collection Saliva samples were obtained twice daily from each subject, at the moments of departure and return of the owner, defining the subject's period of isolation. Twice-daily sampling was done due to the circadian nature of cortisol concentrations inside the body, causing concentrations to fluctuate throughout each day.

Saliva samples were obtained by the experimenter by placing an absorbing dental roll (Nobadent) between the cheek and the jawline of the dog's mouth, preventing the dog from chewing on the dental roll or swallowing it. The dental roll remained in position for one minute before being removed and placed inside 
a $10 \mathrm{ml}$ syringe. The sample was then diluted with $2 \mathrm{ml}$ of phosphate buffered saline (PBS) and pressed into a labeled test tube, which was then sealed and stored for a maximum of 4 weeks at $-18{ }^{\circ} \mathrm{C}$ degrees before being analyzed in a laboratory.

Cortisol concentration determination Cortisol concentrations in the samples were assessed using an enzyme-linked immunosorbent assay (ELISA) cortisol test. After a standardized sequence of steps, reactions produce a color signal in the sample substrates. Light absorbance at $450 \mathrm{~nm}$ is then read by a microplate reader for each sample. Higher cortisol concentrations result in less color intensity in the sample reaction product, and lower optical absorbance values (expressed as optical units).

Although the ELISA cortisol test is used to assess cortisol levels in canines frequently, a preliminary test was performed to determine whether the cortisol concentrations of the samples taken from dogs would fall within the detectable range of the ELISA cortisol test [8]. This confirmed that the cortisol concentrations in the preliminary test samples were detectable within the calibration range of $0.4-1.7 \mathrm{~nm} / \mathrm{mg}$.

Ethograms and qualitative notes Continuous video and audio observations were recorded during the period wherein the subjects were isolated. Cortisol concentrations give an objective indication of the amount of stress experienced by a canine during the test. However, the observed cortisol concentration does not discriminate between positive arousal (excitedness) and negative arousal (anxiety). In order to add context to the cortisol concentrations, video/audio observations of the subjects behavior are analyzed [1].

Behavioral observations were quantified through a focal animal sampling ethogram also used by Scaglia et al. to analyze home alone dogs [40], modified to incorporate interactions with the digital interactive game. An ethogram was created for the first 30 minutes of every hour of video/audio recording, to gain a representative sample of subject behavior. Supporting qualitative notes and observations were made in addition to the quantified ethogram data. This was done to ensure that the proper context was attributed to the subjects' observed behaviors for interpretation.

\section{Results}

\subsection{Subjects}

For the study 3 canine subjects were tested in their natural domestic situation in the Groningen province of The Netherlands during Summer 2014. Table 2 shows key characteristics of the subjects, the isolation duration, and the daily times at which saliva samples were collected. 
Table 2. Overview of subjects, experimental conditions and sample collection characteristics

\begin{tabular}{c|c|c|c}
\hline \multicolumn{4}{c}{ Subjects } \\
\hline Name & Isa & Tommie & Rosie \\
Breed & White Swiss Shepherd & English Springer Spaniel & Australian Shepherd \\
Gender & $\mathrm{F}$ & $\mathrm{M}$ (neutered) & $\mathrm{F}$ \\
Age (in years) & 6 & 5 & 5 \\
Cohabiting animals & 2 cats & none & $1 \mathrm{dog}$ \\
\hline \multicolumn{3}{c}{ Stimulation and sampling conditions per subject } \\
\hline Stimulated condition & $21 / 7-25 / 7 / 2015$ & $28 / 7-1 / 8 / 2015$ & $11 / 8-15 / 8 / 2015$ \\
Unstimulated condition & $28 / 7-1 / 8 / 2015$ & $21 / 7-25 / 7 / 2015$ & $4 / 8-8 / 8 / 2015$ \\
Daily isolation hours & $3(10: 30-13: 30 \mathrm{~h})$ & $2(11: 10-13: 10 \mathrm{~h})$ & $1(20: 00-21: 00 \mathrm{~h})$ \\
\hline
\end{tabular}

\subsection{Salivary cortisol analysis}

The ELISA cortisol test was performed on a duo set of calibration samples and 60 saliva samples obtained from the subjects during the experiment. Sample optical absorbance measurements (in optical units) are usually converted to cortisol concentrations $(\mathrm{ng} / \mathrm{ml})$ by creating a standard curve from the double sets of calibration samples, for which the cortisol concentrations are known. However, rather than converting to the cortisol concentrations we use optical absorbance measures in our data analysis. Converting the absorbance measures to cortisol concentrations introduces noise. Moreover, we are interested in daily withinsubject differences in cortisol levels across experimental conditions, for which absorbance measures suffice as a proxy. The range of absorbance measures encountered in samples is 2.182-2.424 optical units. Between-subject comparisons of cortisol concentrations were not made, because levels are known to differ between individuals and breeds and fluctuate throughout the day, whereas subjects were tested at different times of day. All statistical analyses assume possible effects in either direction (two-tailed) and accept significance if $p<0.05$. To keep the text legible, the unstimulated condition is referred to as control and the stimulated condition as experimental. All results are summarized in Table 3.

Table 3. P-Values of T-tests comparing mean absorbance measurements, across various conditions for pooled and individual subjects. Significant P-values in boldface

\begin{tabular}{c|l|l|l|c|c|c|c}
\hline Data & T-test compares & Pooled & Isa & Tommie & Rosie \\
\hline Control & $\mathrm{P}$ & Pre & Post & $\mathbf{0 . 0 3 5}(-)$ & $0.607(-)$ & $0.077(-)$ & $0.371(-)$ \\
Exp. & $\mathrm{P}$ & Pre & Post & $0.376(-)$ & $0.610(-)$ & $0.303(-)$ & $0.828(+)$ \\
Pre & $\mathrm{U}$ & Control & Exp. & $0.169(+)$ & $0.646(-)$ & $\mathbf{0 . 0 3 2}(+)$ & $0.305(+)$ \\
Post & $\mathrm{U}$ & Control & Exp. & $\mathbf{0 . 0 3 4}(+)$ & $0.251(-)$ & $\mathbf{0 . 0 1 8}(+)$ & $\mathbf{0 . 0 2 4}(+)$ \\
\hline
\end{tabular}

(Control): Unstimulated isolation; (Exp.): Stimulated isolation;

(Pre): Pre-isolation; (Post): Post-isolation; (P): Paired;

(U): Unpaired; (-): Decrease; $\quad(+)$ : Increase 
Under the control condition, and pooled over all subjects, individual absorption values show a small (-0.031) but statistically significant mean daily decrease (paired t-test, $p=0.035, d f=14$ ), i.e. increase of cortisol concentrations. However, under the experimental condition, a smaller mean daily decrease $(-0.011)$ in individual absorption values is found, which is not significant (paired t-test, $p=0.376, d f=14$ ). These findings correspond to our hypothesis that stimulation lessens the mean individual daily increase in cortisol hormone, when compared to the control condition, in fact mitigating its statistical significance. A comparison of pre-isolation measurements between the control and experimental conditions shows a non-significant small different between means of 0.019 (unpaired two-sample t-test, $p=0.169, d f=26$ ), where the experimental condition yields the highest absorbance value. However, when comparing post-isolation samples, the control and experimental conditions shows a small but statistically significant difference between means (unpaired two-sample t-test, $p=0.034$, $d f=26)$. This corresponds to our hypothesis that stimulation decreases the post-isolation cortisol hormone levels compared to the control condition. This effect is not found when comparing pre-isolation samples, as would be expected, because they are independent of experimental conditions.

When comparing means of daily change in absorbance values between control (-0.031) and experimental (-0.011) conditions, pooled over all subjects, the difference is non-significant (unpaired two-sample t-test, $p=0.267, d f=28$ ). Although stimulation appears to lessen the daily increase of cortisol concentrations over all subjects, we cannot reject the null-hypothesis that the true means daily differences in cortisol concentrations are in fact equal when comparing the control and experimental conditions.

Since in theory the latter lack of significance could be caused by pooling of data over subjects, we performed analyses per subject. In short, for two subjects (Tommie, Rosie) post-isolation absorbance values are significantly higher in the experimental condition. Although this corresponds to our hypothesis, it does not hold for subject Isa. Moreover, subject Tommie also shows significantly higher experimental pre-isolation absorbance values, something that cannot be explained through our hypothesis.

\subsection{Ethograms and qualitative notes}

Quantified ethogram scores describing exhibited behaviors were converted to fractions over sample periods, representing the relative occurrences of said behavior. The amount of time subjects were visible in the video was noted. Behaviors exhibited in video view were noted, as were audio cues when subject was outside video view. Detailed ethogram data is reported in [13].

Subject Isa was barely observed on video during stimulated isolation. She was not in proximity of the digital interactive game (to which the camera was aimed). Based on the observations made during her unstimulated trials, Isa was most likely performing passive behavior at a location outside the scope of the camera. Based on collective observations made we assess that Isa exhibits little or no stress-associated behavior during isolation. 
Subject Tommie exhibited more barking, whining and howling during unstimulated isolation compared to stimulated isolation, whereas more locomotion and passive behavior was observed during stimulated isolation. Barking, whining and howling are often perceived as stress related behaviors whereas passive behavior (as exhibited here) is perceived as a calm state of mind. Tommie exhibited slightly more exploratory behavior during stimulated isolation, which is often associated with a perceived sense of safety in the canine. Overall, ethogram data suggest that Tommie shows more stress-associated behavior during unstimulated isolation and more calm behavior during the stimulated isolation.

Subject Rosie performed more barking, whining, locomotion and alert behavior during unstimulated isolation compared to stimulated isolation. When stimulated, Rosie did not howl, exhibited more explorative behavior and appeared more in video, indicating she was in proximity of the game about $90 \%$ of the time. Overall, ethogram data suggest that Rosie performed more stressassociated behaviors during unstimulated isolation, whereas little or no stressassociated behavior was shown in stimulated isolation.

\subsection{Game oriented behaviour}

Subject Isa showed explorative behavior towards the game about $13 \%$ of the time analyzed. She did not play the game, however, possibly because she was not taught to play the game before stimulated isolation. Furthermore, she did not follow any training courses (agility, flyball or obedience training). She did know basic commands, but had never worked with buttons prior to stimulated isolation. Not knowing how to play the game might have resulted in a loss of interest.

Subject Tommie showed slightly more interest in the game and explorative behavior towards the dispenser by sniffing it. He did not play the game, probably because of similar reasons as described for subject Isa. Tommie does know basic commands, but at the time of testing was not involved in any training. He had not been taught to press a button. Both Isa and Tommie showed much interest in the game in the presence of humans.

The fraction of time subject Rosie was in camera view, and thus in proximity of the game, is $91 \%$ percent. She showed interest in the dispenser and even managed to displace it so as to reach the treats inside. Commonly, Rosie performed this behavior after an attempt to play the game while it was not active (no sound was made). This behavior led us to adjust the game settings on the last day of stimulated isolation. Rosie showed more interest in the game than Isa and Tommie and is the only subject who managed to successfully play the

game. Rosie knows basic commands, and was engaged in agility, obedience and doggy dance training. Next to that she had worked with a button before.

\section{Conclusions and discussion}

Our experiment explores the hypothesis that the presence of a digital interactive game, with which a dog can play without the need of human interference, 
can reduce the stress response in dogs when left home alone by their owners. Combining behavioral (ethogram) observations with physiological measurement of cortisol hormone concentrations in canine saliva we observed the following.

(1) Dogs that showed behavioral patterns associated with anxiety in unstimulated isolation showed less behavioral anxiety patterns while stimulated, and these anxiety decreases are confirmed by statistically significant differences in post-isolation cortisol ELISA measurements of these dogs $(n=10$ per dog, 2 dogs). Such decrease in behavioral and physiological anxiety indicators is not observed for a dog that showed no anxiety indicators in unstimulated isolation. However, even when the post-isolation physiological measures $(n=30)$ are pooled over all subjects, including those of the "non-affected" dog, the decrease in physiological anxiety measures remains statistically significant. From these results we are satisfied to accept our main hypothesis to hold for dogs that show behavioral signs of anxiety during normal (i.e. without digital interactive game) isolation. For dogs that show no behavioral signs of anxiety during isolation, the hypothesis is rejected.

(2) Paired within-subject comparison ( $n=15$ pairs) of pre- and post-isolation physiological measures showed a significant increase in physiological anxiety during normal isolation. This increase is much smaller and no longer significant ( $n=15$ pairs) for isolation with the interactive game. This indicates a relevant decrease in physiological anxiety measures when introducing the interactive game. However, comparing daily isolation-induced changes of cortisol ELISA measures between normal isolation $(n=15)$ and isolation with the interactive game $(n=15)$ shows that the decrease exists, but is not significant. Therefore, we cannot with $95 \%$ confidence reject the alternative hypothesis (null-hypothesis) that the daily change in canine physiological stress indicator induced by isolation is similar in the presence of our interactive game. The observed lowered daily increase in physiological anxiety measure could be caused by chance.

(3) Behavioral analysis (and to some degree physiological measures) show that the game is not suitable for every type of dog. A dog that is not stressed or bored when isolated, without the urge to be active or cognitively challenged, will not be attracted to engaging in digital interactive gameplay. A dog in need of cognitive stimulation and known to be active might experience benefits from being able to play the game during isolation. Dogs with such personality traits often descended from breeds such as Border Collies, Australian Shepherds, Malinois and Dutch Shepherds. From our experience, it appears that dogs with these traits develop separation anxiety in particular. A digital interactive game may not only be used to treat separation anxiety, but possibly also as a method to train such dogs for preventing the development of separation anxiety.

That said, we find substantial support from combined quantified behavioral data and physiological stress indicators to conclude that positive effects of the digital interactive game during canine isolation exist and were found. Aspects that may have affected our results should be identified also. Firstly, the small number of subjects $(3 \mathrm{dogs})$ limits the expressive power of our physiological data, even though substantial data per subject was gathered (20 physiological 
measures per dog). Cost-efficiency and practical constraints have played part in this limitation. Secondly, the variances in subject characteristics (e.g. breed, training experience, and exhibited isolation anxiety) complicate generalization of our results. Thirdly, the unfortunate coincidence of our testing with common summer holidays in The Netherlands led to shortened durations of isolation periods. We speculate that longer isolation might strengthen the found effects of digital interactive gameplay on physiological measures of anxiety.

Concerning design of interactive digital games for canines, we conclude the following from our results. Firstly, our game was designed not towards commercial viability, but to test our hypothesis. For general application its robustness could be improved and setting of specific gameplay parameters should be further explored. Secondly, one could argue that dispensing of treats can alter the psychological state of dogs, whereby it did not occur in control conditions. Treats were regarded as part of the complete interactive game experience. Thus their effect on the psychological state of our subjects is considered an effect of interacting with the game as a whole. Thirdly, this reasoning about treats must be generalized; further deconstructing game elements while testing them individually would provide valuable insights as to which game components have what effect.

From the effects observed we conclude that interactive digital gaming without human interference has the potential to improve the quality of life for home alone dogs. Hopefully our work contributes to further study and deployment of interactive digital games for improvement of animal welfare. Moreover, we have shown that physiological indicators combined with behavioral observations provide a strong basis for evaluating effects of digital interactive entertainment on animals. We look forward to interactive entertainment researchers embracing this method. Finally, we are confident to have shown that animals are users worthy of designing intelligent technologies for interactive entertainment for.

\section{Acknowledgement}

We gratefully relied on laboratory support by Petra Bakker (Leiden University), discussion with animal health and welfare specialist Marc Bracke (Wageningen University), and the dog owners voluntarily sharing their time, dogs and homes for this study.

\section{References}

1. Abrantes, R.: Dog language. Wakan Tanka Publishers (1997)

2. Aslaksen, S. and Aukrust, K.: Hundens adferd når den er hjemme alene. Master Thesis, Institutt for Husdyr- og Akvakulturvitenskap, Norway (2003)

3. Beerda, B. et al.: The use of saliva cortisol, urinary cortisol, and catecholamine measurements for a noninvasive assessment of stress responses in dogs. Hormones and Behavior, 30.3, 272-279 (1996)

4. Beerda, B. et al.: Behavioural and hormonal indicators of enduring environmental stress in dogs. Animal Welfare, 9.1, 49-62 (2000) 
5. Campbell, S. A. et al.: Some effects of limited exercise on purpose-bred beagles. American Journal of Veterinary Research, 49.8, 1298-1301 (1988)

6. Coppola, C. L., Grandin, T., and Enns, R. M.: Human interaction and cortisol: can human contact reduce stress for shelter dogs? Physiology \& Behavior, 87, 537-541 (2006)

7. Coren, S.: How dogs think: understanding the canine mind. Simon and Schuster, New York (2005)

8. de Weerth, C. et al.: Measurement of cortisol in small quantities of saliva. Clinical Chemistry, 49.4, 658-660, (2003)

9. Dognition: see the world through your best friends eyes, www.dognition.com

10. Dreschel, N.A. and Granger, D.A.: Methods of collection for salivary cortisol measurement in dogs. Hormones and Behavior, 55.1, 163-168 (2009)

11. Evans, M.: Dogs: Their secret lives. Channel 4 Television (2013)

12. Gácsi, M. et al.: Explaining dog wolf differences in utilizing human pointing gestures: selection for synergistic shifts in the development of some social skills. PLoS One, 4.8 (2009)

13. Geurtsen, A.: An experiment in animal welfare informatics: effects of digital interactive gameplay on the psychological welfare of home alone dogs. Master of Science Thesis, Media Technology program, Leiden University (2014)

14. Grooten, E.: Playing with pigs: researching the complex relationship between pigs and humans through game design, www.playingwithpigs.nl

15. Hare, B. et al.: The domestication hypothesis for dogs' skills with human communication: a response to Udell et al (2008) and Wynne et al (2008). Animal Behaviour, 79, e1-e6, (2010)

16. Hare, B. and Woods, V.: The genius of dogs: discovering the unique intelligence of man's best friend. Oneworld Publications, London (2013)

17. Hawkley, L. C. and Cacioppo, J. T.: Loneliness matters: a theoretical and empirical review of consequences and mechanisms. Annals of Behavioral Medicine, 40, 218227 (2010)

18. Hekman, J.P., Karas, A. Z., and Dreschel, N. A.: Salivary cortisol concentrations and behavior in a population of healthy dogs hospitalized for elective procedures. Applied Animal Behaviour Science, 141.3, 149-157 (2012)

19. Hertz, G.: Cockroach controlled mobile robot, www.conceptlab.com/roachbot

20. Herzing, D.: Could we speak the langauge of dolphins? Video, TED2013 (2013)

21. Hirskyj-Douglas, I. and Read, J. C.: Animal Computer Interaction Design. ACMW'14, Manchester, UK (2014)

22. Holze, R. and Shimoyama, I.: Locomotion control of a bio-robotic system via electric stimulation. Proc IEEE/RSJ Int Conf on Intelligent Robots and Systems, 3, 1514-1519 (1997)

23. Animal hospital of North Asheville: What's your dog's style? Breeds, toys and play styles, www.ahna.net/what-your-puppys-style-breeds-toys-and-play-styles

24. Jensen, P.: The behavioural biology of dogs. Cabi Publishing, UK (2007)

25. Lamers, M. H. and van Eck, W.: Why simulate? Hybrid biological-digital games. Applications of Evolutionary Computation. LNCS, vol. 7248, pp. 214-223 (2012)

26. Maharbiz, M. M. and Hirotaka, S.: Cyborg beetles. Scientific American, 303.6, 94-99 (2010)

27. Mancini, C.: Animal-computer interaction: a manifesto. Interactions, 18, 69-73 (2011)

28. Mancini, C. and Zamansky, A.: Charting unconquered territories: intelligent systems for animal welfare. 40th Annual Convention of the Society for the Study of Artificial Intelligence and the Simulation of Behaviour, pp. 181-182 (2014) 
29. Mankoff, D. et al.: Supporting interspecies social awareness: using peripheral displays for distributed pack awareness. Proc UIST '05, pp. 253-258 (2005)

30. Miklósi, A. et al:: A simple reason for a big difference: wolves do not look back at humans, but dogs do. Current Biology, 13.9, 763-766 (2003)

31. Miklósi, A. et al:: A comparative study of the use of visual communicative signals in interactions between dogs (canis familiaris) and humans and cats (felis catus) and humans. Journal of Comparative Psychology, 119.2, 179 (2005)

32. Miklósi, A. and Soproni, K.: A comparative analysis of animals' understanding of the human pointing gesture. Animal Cognition, 9.2, 81-93 (2006)

33. Miller, P. E. and Murphy, C. J.: Vision in dogs. Journal of the American Veterinary Medical Association, 207.12, 1623-1634 (1995)

34. Noz, F. and An, J.: Cat cat revolution: an interspecies gaming experience. Proc SIGCHI Conf on Human Factors in Computing Systems, pp. 2661-2664 (2011)

35. Overall, K. L. and Dyer, D.: Enrichment strategies for laboratory animals from the viewpoint of clinical veterinary behavioral medicine: emphasis on cats and dogs. Ilar Journal, 46.2, 202-216 (2005)

36. Paarsovaara, S. et al.: The secret life of my dog: design and evaluation of pawtracker concept. Proc 13th Int Conf on Human Computer Interaction with Mobile Devices and Services, pp. 231-240 (2011)

37. Rehn, T. and Keeling, L. J.: The effect of time left alone at home on dog welfare. Applied Animal Behaviour Science, 129, 129-135 (2011)

38. Resner, B. I.: Rover@home: computer mediated remote interaction between humans and dogs. MSc Thesis, Media Arts and Sciences program, Massachusetts Institute of Technology (2001)

39. Savage-Rumbaugh, S., Fields, W. M., and Taglialatela, J. P.: Ape consciousnesshuman consciousness: a perspective informed by language and culture. American Zoologist, 40.6, 910-921 (2000)

40. Scaglia, E. et al:: Video analysis of adult dogs when left home alone. Journal of Veterinary Behavior: Clinical Applications and Research, 8.6, 412-417 (2013)

41. Skinner, B. F.: Pigeons in a pelican. American Psychologist, 15:28 (1960)

42. Talwar, S. K. et al.: Behavioural neuroscience: rat navigation guided by remote control. Nature, 417.6884, 37-38 (2002)

43. Tan, R. T. K. C., Cheok, A. D., and Teh, J. K. S.: Metazoa ludens: mixed reality environment for playing computer games with pets. International Journal of Virtual Reality, 5.3, 5358 (2006)

44. Tuber, D. S. et al.: Behavioral and glucocorticoid responses of adult domestic dogs (canis familiaris) to companionship and social separation. Journal of Comparative Psychology, 110, 103-108 (1996)

45. van Eck, W. and Lamers, M. H.: Animal controlled computer games: playing pacman against real crickets. Harper, R., Rauterberg, M., Combetto, M. (eds.) ICEC 2006. LNCS, vol. 4161, pp. 31-36. Springer, Heidelberg (2006)

46. van Eck W. and Lamers, M. H.: Hybrid biological-digital systems in artistic and entertainment computing. Leonardo Journal of Arts, Sciences and Technology, 46, $151-158$ (2013)

47. Vestrum, I. G.: Aleneatferd hos hunder som lever i en gruppe. Master Thesis, Husdyrvitenskap program, Institutt for Husdyr- og Akvakulturvitenskap (2009)

48. Wingrave, C. A. et al.: Early explorations of CAT: canine amusement and training. Proc CHI EA, pp. 2661-2670 (2010)

49. Wolfe, T. L.: Policy, program and people: the three p's to well-being. J.A. Mench, L. Krulisch (eds.) Canine Research Environment. Scientists Center for Animal Welfare, Bethseda, MD, pp. 41-47 (1990) 